\title{
Research on Torsional Property of Body-In-White Based on Square Box Model and Multiobjective Genetic Algorithm
}

\author{
Yanmei Meng ${ }^{D},{ }^{1}$ Yuan Liang, ${ }^{1}$ Qinchuan Zhao, ${ }^{1}$ and Johnny Qin ${ }^{2}$ \\ ${ }^{1}$ School of Mechanical Engineering, Guangxi University, Nanning 530004, China \\ ${ }^{2}$ Mineral Resources, Commonwealth Scientific and Industrial Research Organization, 1 Technology Court, \\ Pullenvale QLD4069, Australia \\ Correspondence should be addressed to Yanmei Meng; gxu_mengyun@163.com
}

Received 10 June 2020; Revised 18 November 2020; Accepted 8 January 2021; Published 25 January 2021

Academic Editor: S. A. Edalatpanah

Copyright (c) 2021 Yanmei Meng et al. This is an open access article distributed under the Creative Commons Attribution License, which permits unrestricted use, distribution, and reproduction in any medium, provided the original work is properly cited.

In order to assess the performance of a vehicle in the conceptual design stage, a square box model was proposed to predict the torsional stiffness and the first-order torsional frequency of Body-in-White. The structure of Body-in-White was decomposed into eight simple structural surfaces, from which a square box model was constructed. Based on the finite element method, modified shear stiffness of each simple structure surface was calculated and the torsional stiffness was obtained. Then, simple structural surfaces of Body-in-White were constructed into an eight degree-of-freedom series spring system to calculate the first-order torsional frequency. Furthermore, a multiobjective genetic algorithm was used to determine the thickness and structural reinforcement of panels with small stiffness, so as to achieve the goal of increasing the stiffness while reducing the mass of the panel. The result shows that the optimal values of thickness are reduced by around 9.9 percent without affecting their performance by the proposed method. Compared to the prediction results obtained with the complicated numerical simulation, the relative error of the square box model in predicting the torsional stiffness is 6.04 percent and in predicting the first-order torsional frequency is 0.95 percent, indicating that the prediction model is effective.

\section{Introduction}

The torsional stiffness and the first-order torsional vibration frequency are the key properties for a car body and they are major concerns in the car body design and its optimization procedure. However, irregular structures, e.g., grooves, protrusions and reinforcing ribs, and so on, make them difficult to accurately calculate by a single method of numerical analysis $[1,2]$. The finite element method (FEM) provided a method to compute the torsional stiffness and the low-order torsional frequency of the car body [3]. However, the overall and partial design of Body-in-White (BIW) needs to be repeatedly improved in the conceptual design stage, which leads to a complicated operation and high calculation cost. Therefore, a quick and convenient method to predict the torsional properties of BIW is desirable. In this paper, a modeling method of the square box model is proposed to accurately predict the torsional stiffness and the first-order torsional frequency of the car body. Within the framework of the square box model, it is convenient to optimize the mass of BIW so as to ensure a lightweight effect via a multiobjective genetic algorithm (MOGA), which is motivated to solve the difficulty as has been stated.

The properties of BIW have been intensively investigated and a number of optimization methods have been developed in the last few decades. Pawlowski and Guy [4] decomposed a car body structure into a simple structure surface (SSS) to analyze the load and its transmission path, which provided a new approach to assess the property of a car body. Nishigaki et al. [5] proposed a breed of computer aided engineering to analyze the firstorder analysis for a car body in conceptual design due to the sophisticated operations. Lyu and Saitou [6] decomposed a BIW to optimize the stiffness of the assembled structures via MOGA, which only considered the 
static property of the structures. Mundo et al. [7-9] proposed an engineering approach to simplify modeling structures so that the NVH optimization of BIW could be performed at the earliest phases of the vehicle design process. Nariman-Zadeh et al. [10] proposed a multiobjective uniform-diversity genetic algorithm to optimize a five-degree-of-freedom (DOF) vehicle vibration model which provided more choices for optimal design. Gao et al. $[11,12]$ proposed the MOGA algorithm to optimize the structures considering dynamic performance. Li et al. $[13,14]$ proposed a novel approach with concept analysis and implicit parameterization modeling method to build an analytical model of BIW so as to solve its multiobjective problem for higher performance. Nangolo et al. [15] established a mathematical model of a nine-DOF vertical vibration system for a body of four-axis trucks and applied modal analysis to differential equations which had the potential to describe a particular research system. Vasudev et al. [16] proposed a multiobjective optimization design framework that was integrated with NSGA-II, CAD, and CFD for the design of autonomous underwater vehicles, and the optimal parameters achieved better requirements. In recent years, Kiani et al. [17] used surrogate-based optimization to optimize the structure of a BIW considering vibration and stiffness requirements. Mitseas et al. [18] solved a multiobjective problem for nonlinear multi-DOF structural systems by genetic algorithm (GA). Qin et al. [19] proposed a method of transfer stiffness matrix and GA to promote the conceptual design of BIW structure. Shan et al. [20] proposed an assembly method of the modular body through decomposing a structure, and then a simulation was conducted to obtain the design variables via the NSGA-II algorithm. Dama et al. [21-23] proposed an improved GA optimizer and MOGA algorithm for shape optimization of a car body. Deng et al. [24-26] applied an evolution algorithm to a complex optimization problem which had proved effective in solving an actual engineering optimization problem. Ferro et al. [27] proposed a new algorithm to design lightweight and stiff structures that exhibited free-form features based on the coupling of geometric shape optimization with topology optimization.

Through the survey and analysis of the literature, we know that most researchers separately investigated the static properties and dynamic properties of BIW, and the relationship between the static and dynamic characteristics is unrevealed. In this paper, a square box model of BIW is constructed based on the method of SSS. And then, an eight-DOF series spring system is established. Using ANSYS Workbench, the modified stiffness of SSS is obtained to predict the torsional stiffness of BIW. The stiffness matrix is made up of the stiffness of each structural surface. Then, the natural frequency of the multi-DOF undamped vibration system is calculated together with the mass matrix to calculate the first-order torsional frequency. The advantage of the square box model is associated with the static properties and dynamic properties of BIW. Furthermore, with increasing stiffness and reducing mass as objectives, the structure of SSS with small stiffness in the square box model is strengthened and optimized by MOGA. The contribution to the proposed method is that the design requirements are related to a multiobjective optimization problem with MOGA under the framework of the square box model. Moreover, it is helpful for designers to find the optimal position of the structural surface which is needed to reinforce without computation of the whole BIW structure. The novel method is proposed to meet the requirements with designation and size optimization without sophisticated operations.

The rest of this paper is organized as follows. Section 2 introduces the method of the square box model. And the novel contribution is highlighted in Section 2.4. Section 3 describes the new optimization method and the verification is applied. The effectiveness of the proposed method is discussed in Section 4. Finally, Section 5 includes the conclusions and future works.

\section{Materials and Methods}

2.1. Modeling a Square Box Model. A four-wheel unmanned ground vehicle (UGV) model for carrying a robot arm is simplified; see Figures $1(\mathrm{a})$ and $1(\mathrm{~b})$. The BIW is a carrier body. According to the body profile, the construction of a square box model is shown in Figure 1(c). In order to reduce the amount of computation, the square box model based on the SSS method should make the structural plane into a rectangular of the rule or close to the rectangle, ignoring the sharp angle features of the structural plane.

The square box is decomposed as the SSS model, as shown in Figure 1(d). The SSS characteristics are defined as follows: simple structural surfaces are rigid in their own plane while they are flexible in other planes; i.e., SSS can withstand the pull, pressure, shear and bending moments, and other loads in their own plane, while cannot withstand the bending moment in an orthogonal plane or an outside plane.

A typical method of calculating the torsional stiffness for the whole car body is applied to a pair of force couples at the point of force on the car body so as to obtain the body torsional angle. The point of force is usually a supported point of suspension such as point $A$ and point $B$ in Figure 1(b). Then, the body is acted on fixing constraints on point $\mathrm{C}$ and point $\mathrm{D}$. The results obtained by the method are used as a standard of the torsional stiffness of the car body.

Refer to [28], two equal point forces that force A and force $B$ are assumed acting on the BIW in the opposite direction; see Figure 1(b). These loads produce a torque of $T=6250 \mathrm{~N} \cdot \mathrm{m}$ in the car body. In this paper, a novel method of calculating the torsional stiffness for the overall car body will be discussed later.

Applying the torsion load $T$ to the whole car body, then the load $Q_{j}(j=1,2, \ldots, 7)$ of each SSS is calculated as shown in Figure 2. In this way, the torque $T$ is broken down to decomposition loads acting on each surface.

Equilibrium and compatibility equations for 8 planes SSS- 1 to SSS- 8 are as follows: 


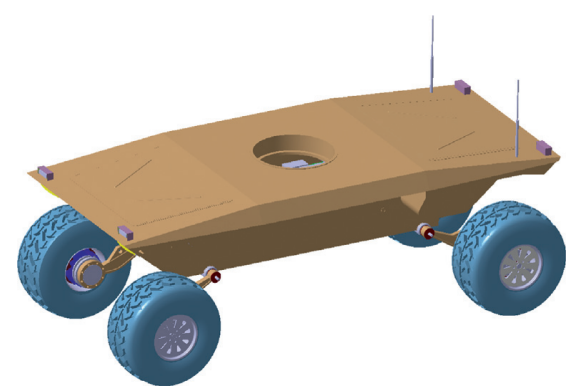

(a)

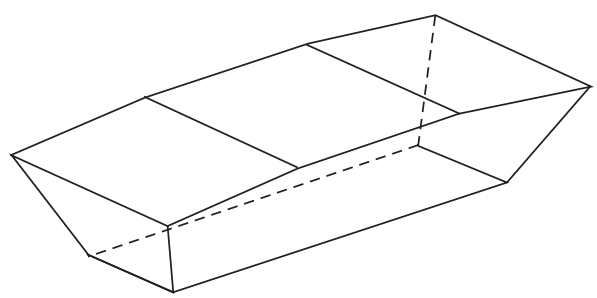

(c)

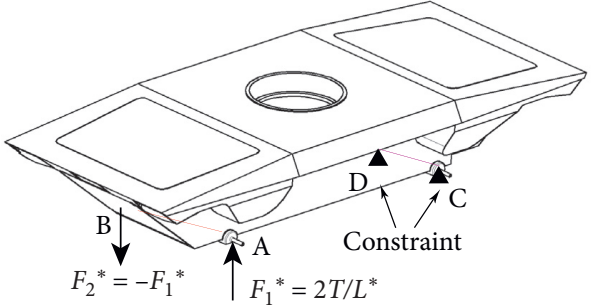

(b)
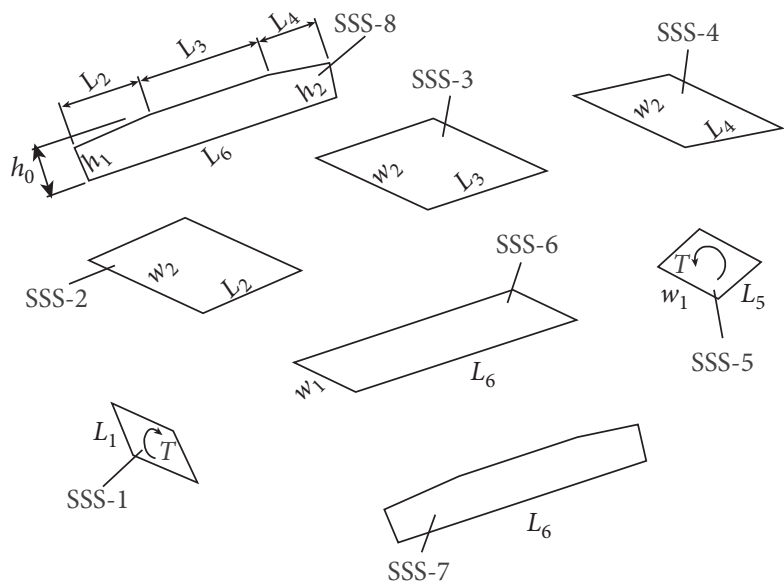

(d)

FIGURe 1: The establishment of the square box model and decomposition of SSS. (a) Model of Four-wheel UGV. (b) Simplified model of BIW. (c) Box model of BIW. (d) Model of SSS.

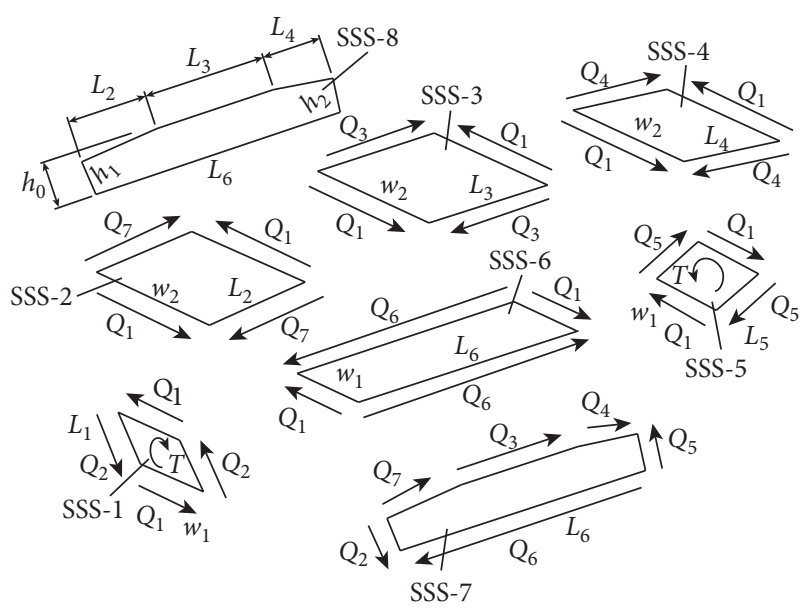

FIGURE 2: The mechanical model of SSS for a BIW.

$$
\left[\begin{array}{ccccccc}
L_{1} & w_{1} & 0 & 0 & 0 & 0 & 0 \\
L_{2} & 0 & 0 & 0 & 0 & 0 & -w_{2} \\
L_{3} & 0 & -w_{2} & 0 & 0 & 0 & 0 \\
L_{4} & 0 & 0 & -w_{2} & 0 & 0 & 0 \\
L_{5} & 0 & 0 & 0 & w_{1} & 0 & 0 \\
L_{6} & 0 & 0 & 0 & 0 & -w_{1} & 0 \\
0 & 0 & \left(h_{0}-h_{1}\right) & d & -L_{6} & h_{1} & 0
\end{array}\right]\left[\begin{array}{c}
Q_{1} \\
Q_{2} \\
Q_{3} \\
Q_{4} \\
Q_{5} \\
Q_{6} \\
Q_{7}
\end{array}\right]=\left[\begin{array}{c}
T \\
0 \\
0 \\
0 \\
T \\
0 \\
0
\end{array}\right],
$$

where $L_{1}=0.78 \mathrm{~m} ; L_{2}=1.05 \mathrm{~m} ; L_{3}=1.30 \mathrm{~m} ; L_{4}=1.05 \mathrm{~m}$; $L_{5}=0.78 \mathrm{~m} ; \quad L_{6}=2.20 \mathrm{~m} ; \quad h_{0}=0.60 \mathrm{~m} ; \quad h_{1}=0.50 \mathrm{~m}$; $h_{2}=0.50 \mathrm{~m} ; w_{1}=0.68 \mathrm{~m} ; w_{2}=1.48 \mathrm{~m} ; T=6250 \mathrm{~N} \cdot \mathrm{m} ;$ and $T$ is directly calculated according to the force outside the body which is the known amount.

Here, $d=\left(h_{2}-h_{1}\right) L_{4}+\left(h_{0}-h_{2}\right) L_{6} / \sqrt{L_{4}^{2}+\left(h_{2}-h_{0}\right)^{2}}$. 
From equation (1), the load of $Q_{j}$ can be obtained as follows: $Q_{1}=4620.25 \mathrm{~N} ; \quad Q_{2}=3891.48 \mathrm{~N} ; Q_{3}=4050.12 \mathrm{~N}$; $Q_{4}=3271.25 \mathrm{~N} ; \quad Q_{5}=3891.48 \mathrm{~N} ; \quad Q_{6}=14947.86 \mathrm{~N}$; $Q_{7}=3271.25 \mathrm{~N}$.

These data provide the basis of the design of torsion strength and cross-sectional shape. Afterward, appropriate cross-sectional shape and dimensions are designed for these loads. Finally, the whole BIW achieves the desired torsion strength.

2.2. Computation Effective Stiffness of SSS. The torsion strength described in Section 2.1 is the basis of a structural design. However, if a BIW is expected to have good handling and good NVH properties, higher requirements are required for a reverse stiffness design [29]. Applying torque $T_{0}$ on the box will yield an angular displacement $\theta$. The torsional stiffness $K$ of the box is as follows:

$$
K=\frac{T_{0}}{\theta}
$$

where $\theta=2 \eta_{\text {body }} / L_{6} ; \eta_{\text {body }}$ is the deformation displacement of the entire car body after acting $T_{0}$ on it.

Then, a mathematical model of torsional stiffness is deduced based on the energy method. Assuming that a shear force is a uniform, and the shear model of a panel is shown in Figure 3(a). Here, a rectangular panel with a size of $(a \times b)$ becomes the diamond-shaped panel of size $\left(a^{\prime} \times b^{\prime}\right)$. The distortion energy is stored in the form of elastic energy.

The energy stored in a single panel is expressed as follows:

$$
e=\int_{\text {Volume }} \frac{\tau \gamma}{2} d V=\frac{Q_{j}^{2}}{2} \frac{a b}{G t},
$$

where $\tau$ is the shear stress, $\tau=Q_{j} / t ; \gamma$ is the shear strain; $\int$ represents full volume integration of the panel; $t$ is the thickness of the panel; $G$ is the shear modulus, $G=\tau / \gamma$; $V$ is the volume of the panel, $V=\mathrm{abt} ;(G t)$ is the shear stiffness of the panel.

The external load applying to the BIW under a torsional condition is converted into the torque of $T_{0}$. It is equal to the shear flow of each panel. Then, the energy stored in all panels is as follows:

$$
E=\frac{1}{2} T_{0} \theta
$$

Then, we get the following:

$$
\begin{aligned}
E= & \frac{Q_{i}^{2}}{2} \cdot\left[\left(\frac{L_{1} w_{1}}{G t}\right)_{\text {SSS }-1}+\left(\frac{L_{2} w_{2}}{G t}\right)_{\text {SSS-2 }}+\left(\frac{L_{3} w_{2}}{G t}\right)_{\text {SSS }-3}+\left(\frac{L_{4} w_{2}}{G t}\right)_{\text {SSS }-4}\right] \\
& \left.+\left(\frac{L_{5} w_{1}}{G t}\right)_{\text {SSS-5 }}+\left(\frac{L_{6} w_{1}}{G t}\right)_{\text {SSS-6 }}+\left(\frac{L_{6} h_{0}}{G t}\right)_{\text {SSS-7 }}+\left(\frac{L_{6} h_{0}}{G t}\right)_{\text {SSS-8 }}\right] .
\end{aligned}
$$

Let the area of panel SSS-1 be denoted as $S_{1}$, then $S_{1}=L_{1} w_{1}$. Similar expressions can be obtained for the other panels.

Then, equation (5) is equivalent as follows:

$$
E=\frac{Q_{j}^{2}}{2} \sum_{j=1}^{8}\left[\frac{S_{j}}{(G t)}\right]
$$

For the convenience of computation, the shear flow of the transformation formula is calculated with the length of panel SSS-1, which is denoted as $w_{1}$. Then, we get the following:

$$
\tau=\frac{T_{0}}{2 w_{1} L_{1}}
$$

According to equations (4), (6), and (7), we get the following:

$$
\theta=T_{0}\left(\frac{1}{2 w_{1} L_{1}}\right)^{2} \sum_{j=1}^{8}\left[\frac{S_{j}}{(G t)}\right]
$$

Furthermore, according to equations (2) and (8), the torsional stiffness of BIW is as follows:

$$
K=\left(2 w_{1} L_{1}\right)^{2} \frac{1}{\sum_{j=1}^{8}\left[S_{j} /(G t)\right] .}
$$

Here, the meaning of the shear stiffness of $(G t)$ in the equation (9) implies that a panel is flat, no pores, no bulge, and no strengthening ribs, etc. In fact, the structural body is mostly a complex structure of the irregular shape. Thus, the value of $(G t)$ needs to be modified. Modified effective shear stiffness is denoted as $(G t)_{\mathrm{EFF}}$. In general, common correction methods include parsing, FEM, and experimental test. For a BIW in the concept design stage, the parsing method and the experimental test method can obtain more reliable and higher precision results. However, the design of the BIW in this stage needs to be continuously improved, leading to the designer with a heavy workload and high cost. The FEM is low cost and it has acceptable numerical accuracy. Therefore, it is suitable for FEM to be used in the conceptual design stage. In this study, the meshed model is imported into ANSYS Workbench to be solved. In the end, the values of $(G t)_{\mathrm{EFF}}$ are calculated for each SSS.

The boundary conditions are applied by the FEM as shown in Figure 3(b). The shear strain, $\gamma$, can be easily obtained. Then, the effective value of $(G t)_{\mathrm{EFF}}$ can be derived as follows:

$$
(G t)_{\mathrm{EFF}}=\tau / \gamma=F b /(\delta a)
$$

Finally, the modified expression of torsional stiffness for $\mathrm{BIW}$ is as follows:

$$
K=\left(2 w_{1} L_{1}\right)^{2} \frac{1}{\sum_{j=1}^{8}\left[S_{j} /(G t)_{\mathrm{EFF}}\right] .}
$$

2.3. Computation of the First-Order Torsional Frequency. Actually, the torsional stiffness and the first-order torsional frequency of a BIW are interrelated. The vibration model of a vehicle is simplified into the vibration model of a multi-DOF system. A mathematical model of equivalent vibration is established based on equation (11). 


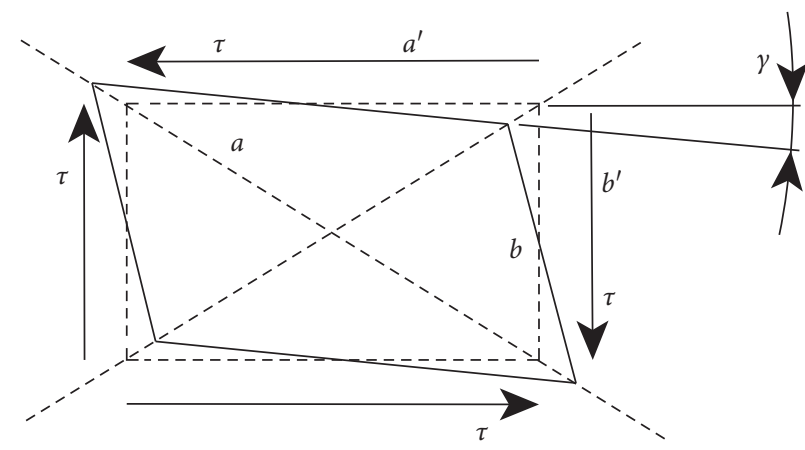

(a)

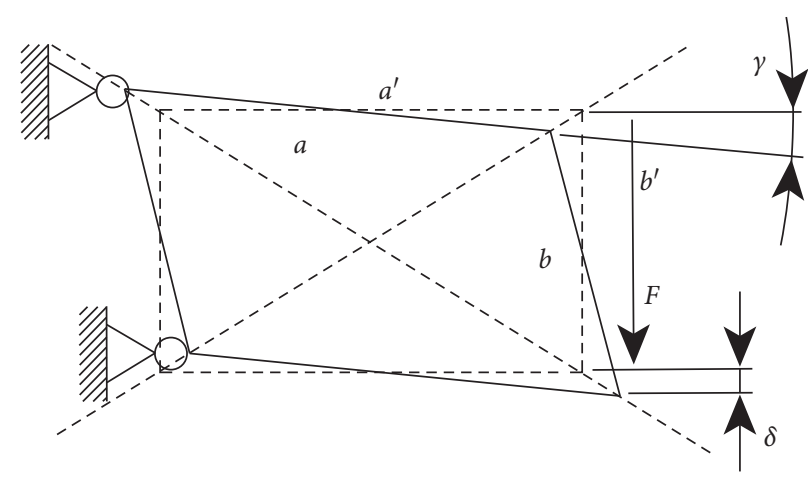

(b)

Figure 3: The single panel of shear forces and boundary conditions. (a) Shear forces of a single panel. (b) Boundary conditions of a single panel.

Take an equivalent transformation of equation (11). Then, we get the following:

$$
K_{j}=\frac{(G t)_{\mathrm{EFF}}}{S_{j}}, \quad j=1,2, \ldots, 8 .
$$

From equations (11) and (12), we get the following:

$$
\frac{1}{K}=\vartheta \sum_{j=1}^{8} \frac{1}{K_{j}}, \quad j=1,2, \ldots, 8,
$$

where $\vartheta$ is the constant coefficient, and $\vartheta=\left(1 / 2 w_{1} L_{1}\right)^{2} ; K_{j} / \vartheta$ represents the stiffness of equivalent spring of SSS.

In fact, equation (13) is an expression of the series spring system. It is used as the equivalent model of the square box model so that the first-order torsional frequency of the BIW is calculated. Due to the material of BIW is steel, the system of damping is very small. Thus, the effect of damping on the vibration system is ignored. As a result, the equivalent model of BIW is constructed as an eight-DOF vibration system without damping, as shown in Figure 4.

The differential equations of motion for a multi-DOF vibration system can be established by the direct method, Lagrange method, or influencing coefficient method, etc. Then, the equations are solved to obtain the natural frequency and the main vibration type of the system.

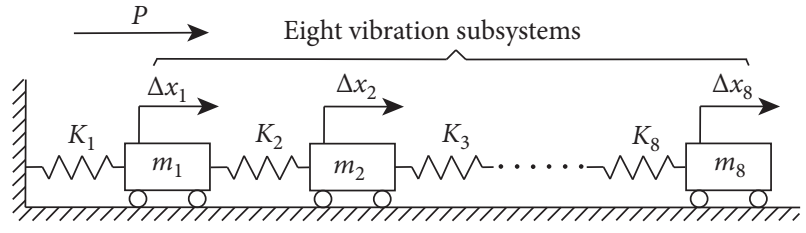

Figure 4: The eight-DOF equivalent vibration model of BIW.

The free vibration equation of a system without damping is as follows:

$$
M \ddot{X}+K X=0,
$$

where $M$ is the mass matrix; $X$ is the displacement matrix; $\ddot{X}$ is the acceleration matrix; and $K$ is the stiffness matrix of the vibration system.

From Figure 4, we get $M$ :

$$
M=\left[\begin{array}{cccccc}
m_{1} & 0 & & \cdots & & 0 \\
0 & m_{2} & \ddots & & & \\
& \ddots & \ddots & 0 & & \vdots \\
\vdots & & 0 & m_{j} & \ddots & \\
& & & \ddots & \ddots & 0 \\
0 & & \ldots & & 0 & m_{8}
\end{array}\right]_{8 \times 8}
$$


By the stiff matrix method, we get $K$ :

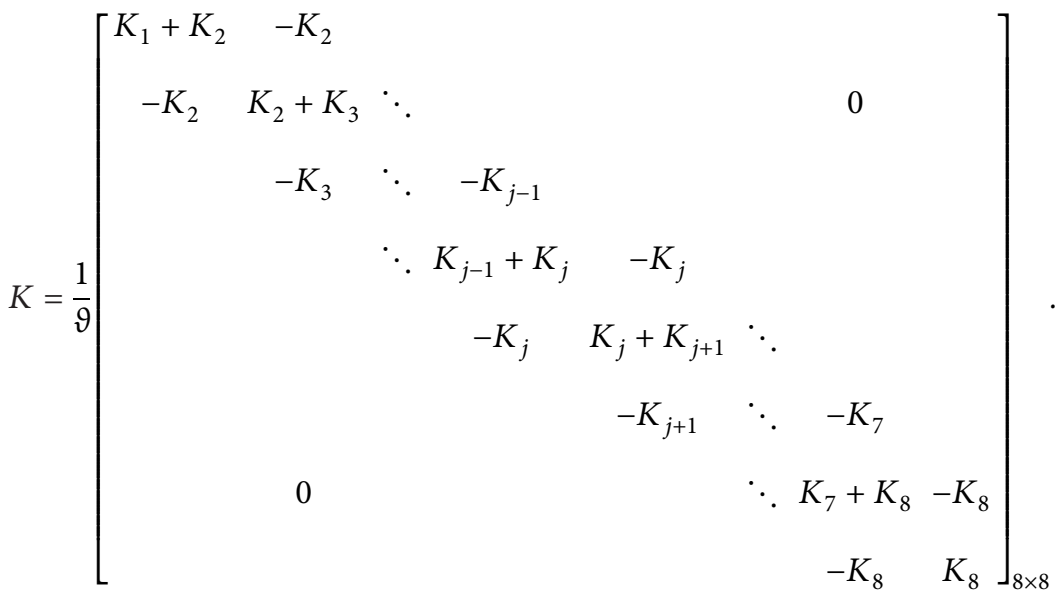

For a multi-DOF free vibration system without damping, the exact solution is obtained by using a real mode analysis method, i.e., the coordinated transformation of the original system to achieve the decoupling of the system. Finally, according to a certain proportion, the solution of the original equation is obtained by superposition of the main modes of each order so as to obtain the natural frequency and the main modes of the vibration system.

The solution of equations (14) to (16) is written as follows:

$$
X=A e^{i \omega_{n} t_{f}},
$$

where $A$ is an amplitude vector for the free vibration of the system, $A=\left[A_{1}, A_{2}, \ldots A_{n}\right]^{\mathrm{T}} ; t_{f}$ is the vibration period; $\omega_{n}$ is the natural circular frequency.

2.4. A New Optimal Strategy. Conceptual design is vital in car body development and it has a great effect on later optimization work. As has been stated, a novel optimal strategy is collaborative with the square box model and MOGA algorithm to solve complex optimization problems. The purpose of building the square box model is that the overall analysis of BIW is divided into the analysis of each SSS, which is simplified the design procedure. Particularly, the computation of torsional stiffness becomes more easily. Moreover, all panels are constructed into the eight-DOF series spring system to calculate the first-order torsional frequency. In general, a new BIW is composed of many small parts and it consumes a lot of time and energy to deal with the boundary condition for the designer. The conventional approach, i.e., single FEM method, is needed to repeatedly compose and decompose into the new BIW. However, the proposed method in this paper has only decomposed the body and can be obtained with the static and dynamic properties on the basis of the optimization process. As for the structure of BIW, the structure exists in a weak position that can be found with the help of the square box model. Then, the weak position is reinforced with increasing weight. Hence, a lightweight optimization to reduce the mass of the car body is proposed. In fact, the strategy of lightweight optimization is to optimize the thickness of panels via size optimization. Likewise, the Latin Hypercube Sampling (LHS) approach is used to represent the uncertainty surrounding the input parameters. Afterwards, the response surface method is adopted to build accurate models in the parameter ranges. Furthermore, a multiobjective optimization method is applied to obtain the optimal thickness within global space. Finally, the optimal parameters are obtained by MOGA.

\section{Numerical Simulation and Optimization}

3.1. Numerical Simulation of SSS. The BIW designed in Figure 5 is taken as an example to illustrate the computation process of the torsional property. The material is $22 \mathrm{SiMn} 2 \mathrm{TiB}$ steel and the original thickness of the steel is $t=2 \mathrm{~mm}$. And the basic properties are shown in Table 1.

We proceed in the following order to get the result of the torsional property. Firstly, a model of BIW is built as Figure 5(a) by CATIA V5. Secondly, the model is decomposed as SSS, see Figure 5(b). Thirdly, the displacement of deformation is calculated for each SSS. Fourthly, the displacement of deformation is used to calculate the value of

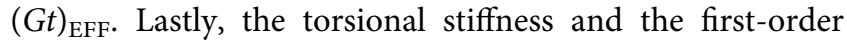
torsional frequency are obtained.

The key to ensuring high accuracy for the prediction model is that the deformation displacement of each panel is correctly calculated. Thus, the $(G t)_{\mathrm{EFF}}$ of SSS-7 is solved to illustrate the solving process of SSS. Similar processes are applied to the other structural surfaces.

According to the force model of the panel in Figure 3, the constraint conditions and the force of $Q_{6}=14947.86 \mathrm{~N}$ which has been calculated in equation (1) are applied to SSS-7. The constraint conditions and the load should be applied to one plane based on Figure 6(a). If the force strictly follows the requirements of the schematic diagram as shown in Figure 3(b), the direction of the force does not affect the calculation results. The direction of smaller stiffness is usually chosen to calculate the $(G t)_{\mathrm{EFF}}$ for a panel.

Considering the BIW as a symmetric body of the longitudinal centre plane, one side of the symmetrical structure is chosen to compute the displacement of deformation. In the environment of ANSYS Workbench 14.5, SSS-7 is meshed and 


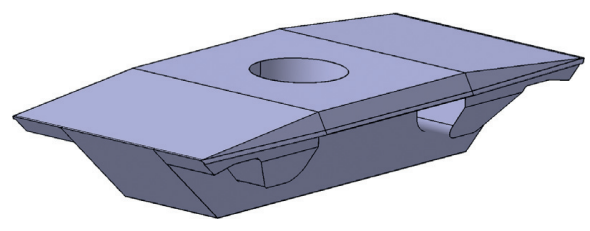

(a)

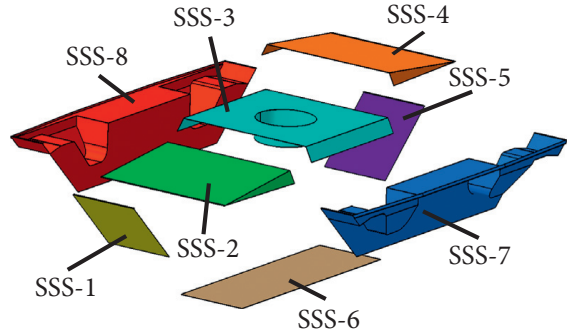

(b)

Figure 5: The structural decomposition of BIW. (a) The model of BIW. (b) The structural decomposition.

TABLE 1: The basic properties of material.

\begin{tabular}{lcccc}
\hline Material & Density, $\mu\left(\mathrm{kg} \cdot \mathrm{m}^{-3}\right)$ & Poisson's ratio, $v$ & Elastic modulus, EX $(\mathrm{Pa})$ & Shear modulus, $G(\mathrm{~Pa})$ \\
\hline 22SiMn2TiB & 7850 & 0.3 & $2.08 \times 10^{11}$ & $8.0 \times 10^{10}$ \\
\hline
\end{tabular}

computed. Figure 6(b) shows the contours of $y$-directional deformation. It is seen that at the middle point $A$ where the force of $Q_{6}$ leads to the displacement $\eta_{7}, \eta_{7}$ is equal to $158 \mathrm{~mm}$. In the same way, $\eta_{j}$ for the remaining surface is also calculated. The value of $S_{j} /(G t)_{\mathrm{EFF}}$ is obtained as shown in Table 2 .

By substituting $S_{j} /(G t)_{\mathrm{EFF}}$ in Table 2 into equation (11), the original torsional stiffness of the BIW can be calculated as $1.83 \times 10^{7} \mathrm{~N} \cdot \mathrm{mm} / \mathrm{rad}$, i.e., $320.154 \mathrm{~N} \cdot \mathrm{m} /\left(^{\circ}\right)$. By substituting $S_{j} /(G t)_{\mathrm{EFF}}$ into equation (16), the value of $K$ is calculated and then the first-order torsional frequency of the original BIW is obtained as $13.232 \mathrm{~Hz}$ from equation (17).

3.2. Optimization of SSS-7. The multiobjective genetic algorithm is a guided random search method that is suitable for solving multiobjective optimization problems as it can explore the diverse regions of the solution space [23]. The solution to MOGA is illustrated by using the Pareto fronts based on the natural biological evaluation principle. It can tackle the convergence problems usually encountered by conventional methods for solving multiobjective problems. The MOGA technique has been widely applied to design and optimize machining parameters. In this study, MOGA is used to optimize the thickness of the BIW structure.

As can be seen, the overall original torsional stiffness of $\mathrm{BIW}$ is far from the engineering design where the shear stiffness of $(G t)_{\mathrm{EFF}}$ is too small, including SSS-2, SSS-3, SSS4, SSS-7, and SSS-8, as shown in Table 2. Therefore, it is necessary to improve the shear stiffness of the panels.

We take SSS-7 as an example to introduce the optimization process of MOGA. Firstly, the structure of SSS-7 is strengthened by adding three channel-shaped steel. Their cross-sectional areas are $40 \mathrm{~mm} \times 20 \mathrm{~mm}$ as shown in Figure 7.

Compared to the original model, SSS-7 has been changed in four places. SSS-7 is needed to parameterize the thickness of these four places, see Figure 7 . The four parameters are set initial values as $x_{1}=3 \mathrm{~mm}, x_{2}=3 \mathrm{~mm}$, $x_{3}=3 \mathrm{~mm}$, and $x_{4}=2 \mathrm{~mm}$, respectively. Due to adding on the new structures, the mass of the structure has been increased. Therefore, it is necessary to optimize the thickness from $x_{1}$ to $x_{4}$ to reduce the mass of the car body as much as possible.

Secondly, in the environment of ANSYS Workbench, the load $Q_{6}$ is acted on SSS-7, and then the deformation displacement of $y$-direction is obtained which is written as Deformation $(x)$. Afterwards, the first-order torsional frequency of SSS-7 is generated which is written as Frequency $(x)$. The typical deterministic constrained multiobjective optimization problem is described as the following mathematical model [30]:

$$
\left\{\begin{array}{l}
\text { Find } x=\left(x_{1}, x_{2}, \ldots, x_{m}\right)^{T}, \\
\min \quad F(x)=\left(F_{1}(x), F_{2}(x)\right)^{T}, \\
\text { s.t. } \quad g_{i}(x)<0, i=1,2, \ldots, p, \\
h_{j}(x)=0, j=1,2, \ldots, q, \\
x_{L} \leq x \leq x_{U},
\end{array}\right.
$$

where $F(x), g(x)$ and $h(x)$ correspond to objective functions, inequality constraints and equality constraints, respectively; $m, p$, and $q$ are the number of corresponding functions; $x$ is the decision vector; here, $F_{1}(x)=$ Deformation $(x)$, and $F_{2}(x)=$ Frequency $(x)$.

A set of Pareto optimal solutions is obtained by the MOGA algorithm. Then, one Pareto optimal solution is selected as the optimal solution of the multiobjective function. The flowchart of the MOGA algorithm is shown in Figure 8 .

Thirdly, the initial population is obtained to produce data samples via the LHS approach. Deformation $(x)$ and Frequency $(x)$ are established as objective functions that are related to the independent variables, i.e., $x_{1}, x_{2}, x_{3}$, and $x_{4}$. The optimization process of the independent variable is iteratively solved as shown in Figure 9. The optimization results are shown in Figure 10. See Figure S1 in the Supplementary Material for comprehensive image analysis of SSS-7 after optimization.

Finally, as can be seen from Figure 10, the population evolution tends to be stable after the 800th iteration which 


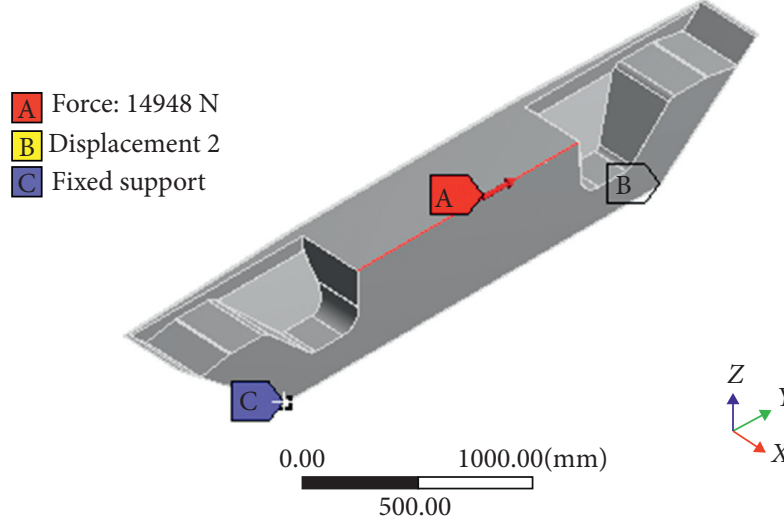

(a)

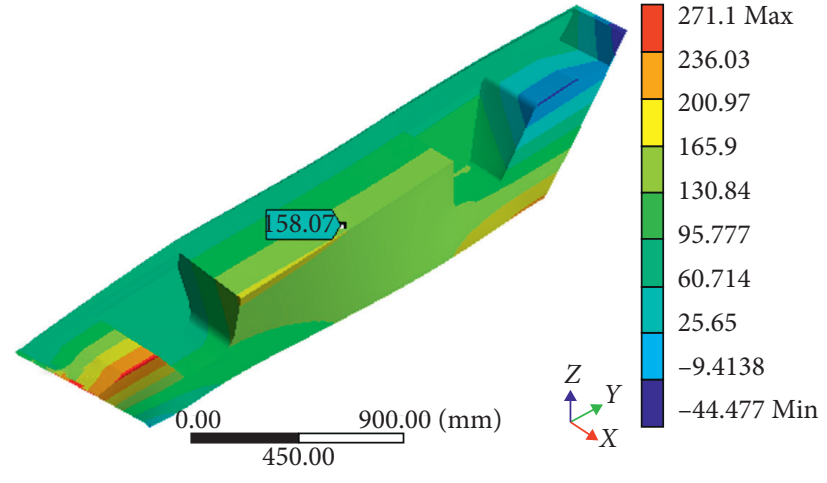

(b)

FIgURE 6: The deformation displacement of SSS-7. (a) The boundary condition of SSS-7. (b) The y-directional deformation of SSS-7.

TABLE 2: The displacement of deformation before optimizations about SSS.

\begin{tabular}{lcccc}
\hline SSS & $\eta_{j}(\mathrm{~mm})$ & Mass, $m_{i}(\mathrm{~kg})$ & $(G t)_{\mathrm{EFF}}\left(\mathrm{N} \cdot \mathrm{mm}^{-1}\right)$ & $S_{j} /(G t)_{\mathrm{EFF}}\left(\mathrm{mm}{ }^{3} \cdot \mathrm{N}\right)$ \\
\hline SSS-1 & 1.31 & 8.53 & 3074.73 & 172.50 \\
SSS-2 & 75.23 & 24.24 & 86.80 & 17944.49 \\
SSS-3 & 27.62 & 34.29 & 191.02 & 10095.56 \\
SSS-4 & 75.23 & 24.24 & 86.80 & 17944.49 \\
SSS-5 & 1.31 & 8.53 & 3074.73 & 172.50 \\
SSS-6 & 2.80 & 23.51 & 510.01 & 6396.91 \\
SSS-7 & 158.07 & 46.02 & 346.73 & 4311.62 \\
SSS-8 & 158.07 & 46.02 & 346.73 & 4311.62 \\
\hline
\end{tabular}

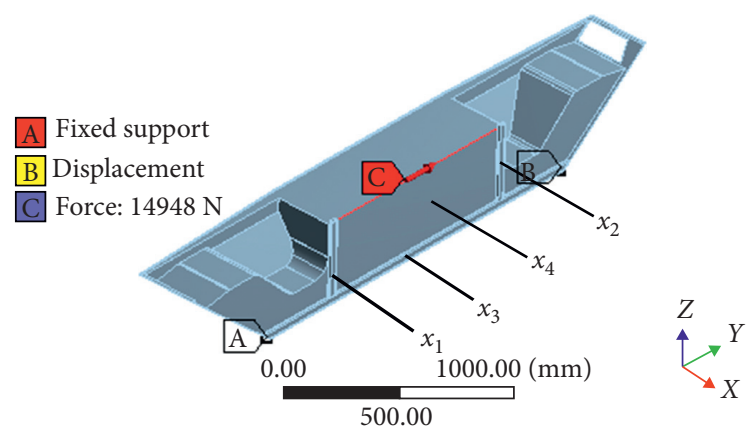

FIGURE 7: Extraction of independent variables about SSS-7.

hardly affects their performance. And then, the optimal solution to Pareto is obtained as shown in Table 3. It is seen that the optimal values are reduced by around $9.9 \%$ for all independent variables from $x_{1}$ to $x_{4}$.

3.3. Computation of $(G t)_{E F F}$ after Optimization. After optimizing all SSS, the original BIW model is improved as shown in Figure 11(a). Then, the structural decomposition of BIW after optimization is shown in Figure 11(b).

In SSS-2 and SSS-4, the ribs are used to strengthen the structures. In order to increase the shear stiffness value of SSS-3, SSS-6, SSS-7, and SSS-8, a closed rectangular frame is constructed inside to further improve the overall torsional stiffness of the BIW. SSS-1 and SSS-5 are cut out some material to install the equipment.

As a comparison, the proposed method is used to calculate the $(G t)_{\mathrm{EFF}}$ and the first-order torsional frequency again for the model after optimization. The displacement and stiffness of eight panels after optimization are shown in Table 4. It is seen that the deformation displacement of SSS-7 has been significantly reduced from $158.07 \mathrm{~mm}$ to $0.2177 \mathrm{~mm}$ after optimization under the same external force of $Q_{6}$. Complete analytical results of optimized SSS are shown in Figure S2 in the Supplementary Material for comprehensive analysis.

By substituting the data of $\left[S_{j} /(G t)_{\mathrm{EFF}}\right]^{\prime}$ in Table 4 into equation (11), the torsional stiffness of the BIW can be 


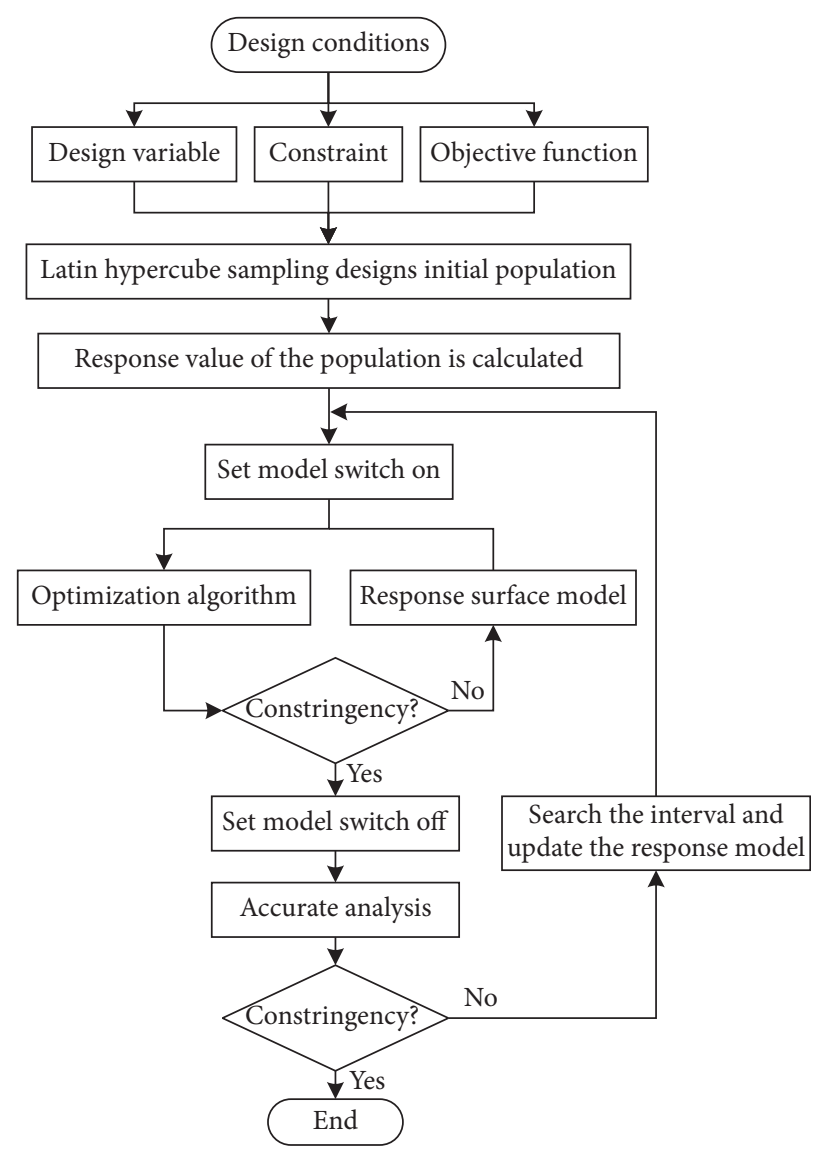

FIgURE 8: The flow of the MOGA algorithm.

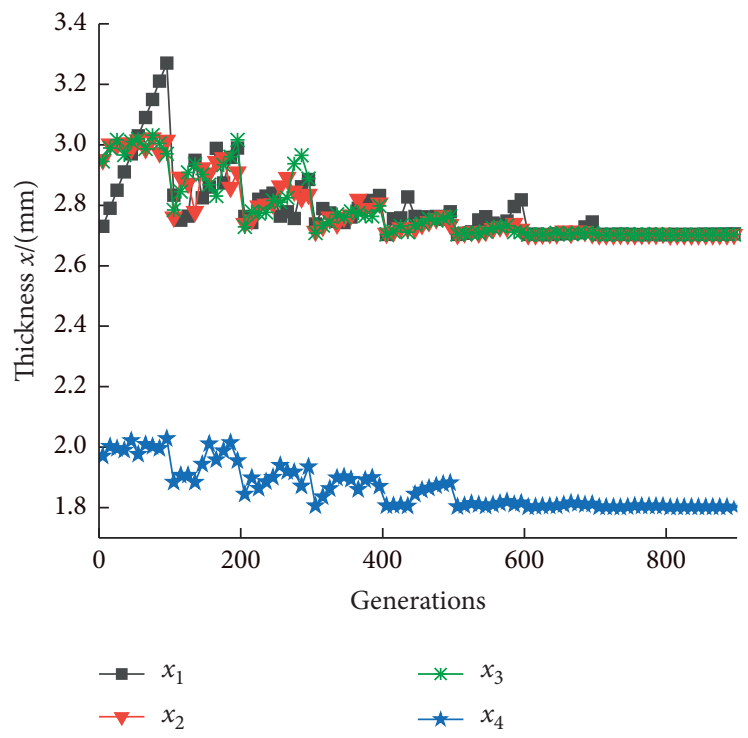

FIGURE 9: Variation of independent variables with the number of generations.

calculated as $7139.785 \mathrm{~N} \cdot \mathrm{m} /\left(^{\circ}\right)$ after optimization, which is more than 20 times of the original one. By substituting the data of $\left[S_{j} /(G t)_{\mathrm{EFF}}\right]^{\prime}$ into equation (16), it can be calculated the value of $K$. And then the first-order torsional frequency of BIW is calculated as $24.987 \mathrm{~Hz}$ after optimization, which is much increased from compared to $13.232 \mathrm{~Hz}$ of the original one. 


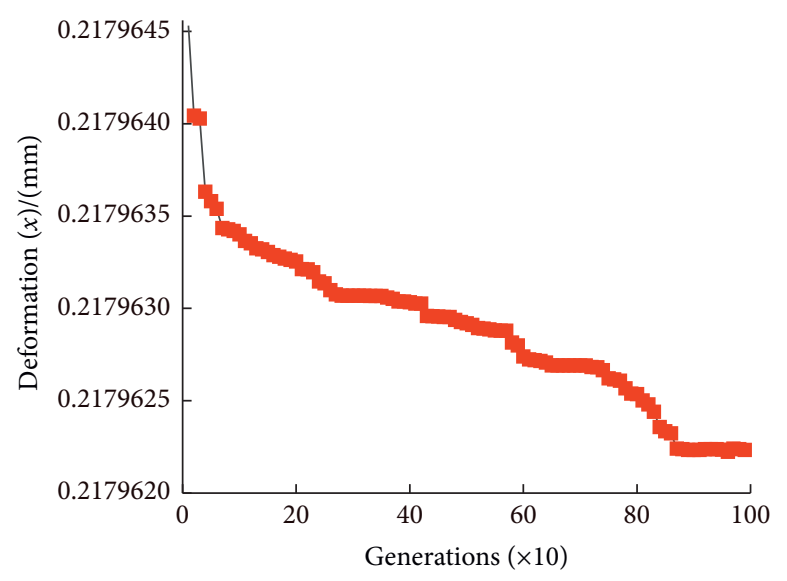

(a)

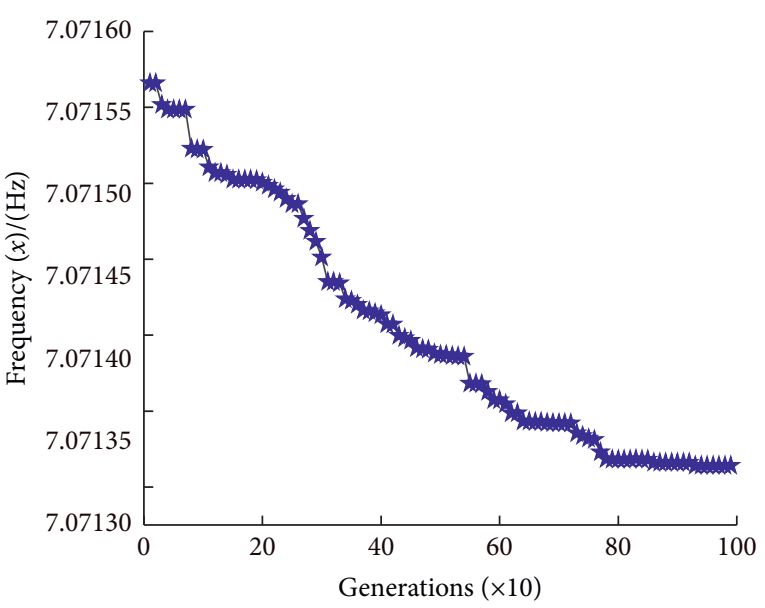

(b)

FIGURE 10: Objective function changes with the number of generations. (a) Deformation changes with the number of generations. (b) Frequency changes with the number of generations.

TABLE 3: Objective optimization variables and results.

\begin{tabular}{lccccc}
\hline Independent variable $(\mathrm{mm})$ & Initial value $(\mathrm{mm})$ & Upper limit $(\mathrm{mm})$ & Lower limit $(\mathrm{mm})$ & Optimal value $(\mathrm{mm})$ & Variation $(\%)$ \\
\hline$x_{1}$ & 3 & 3.3 & 2.7 & 2.7029948 & -9.90 \\
$x_{2}$ & 3 & 3.3 & 2.7 & 2.7005521 & -9.98 \\
$x_{3}$ & 3 & 3.3 & 2.7 & 2.7025491 & -9.91 \\
$x_{4}$ & 2 & 2.2 & 1.8 & 1.8018520 & -9.91 \\
\hline
\end{tabular}

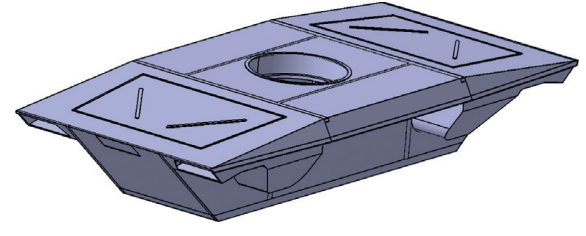

(a)

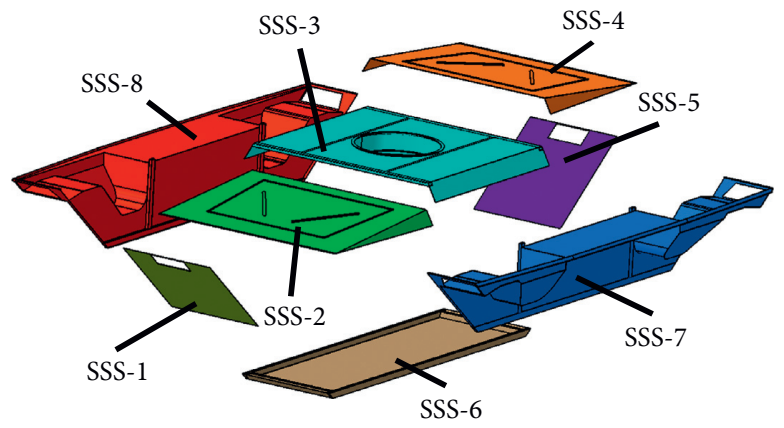

(b)

FIgURE 11: The structural decomposition of BIW. (a) The model of BIW after optimization. (b) The structural decomposition of BIW after optimization.

3.4. Verification of the Whole BIW. In order to verify the reliability of the computation results obtained from the square box model, the following numerical simulation based on FEM is conducted on the whole BIW to directly obtain the torsional displacement deformation and the first-order torsional frequency as shown in Figure 12.

The optimized model is chosen as the calculated object; see Figure $12(\mathrm{a})$. The torque $T=6250 \mathrm{~N} \cdot \mathrm{m}$ is applied to the position of SSS-1 to calculate the torsional displacement. Afterwards, the torsional stiffness of the whole BIW is calculated. After the optimization, the torsional displacement, $\eta_{\text {body }}{ }^{\prime}$, is shown in Figure 12(b). The position of the torsional displacement is around a point of the wheel axis. Then, $\eta_{\text {body }}{ }^{\prime}$ is equal to $17.824 \mathrm{~mm}$ and it is substituted into equation (2) to calculate the torsional stiffness. Finally, the torsional stiffness of the whole BIW is calculated as $3.858 \times 10^{8} \mathrm{~N} \cdot \mathrm{mm} / \mathrm{rad}$, i.e. $6733.394 \mathrm{~N} \cdot \mathrm{m} /\left(^{\circ}\right)$. The first-order torsional frequency is $24.752 \mathrm{~Hz}$ as shown in Figure 12(c).

\section{Results and Discussion}

The torsional stiffness and the first-order torsional frequency are directly obtained by FEM in Section 3.4 as the standard value. In this section, the comparison between the proposed model and the FEM is conducted. Table 5 shows the results that are obtained by the two methods for the same optimized BIW. 
TABLE 4: The calculated results of $(G t)_{\mathrm{EFF}}$ after the optimizations.

\begin{tabular}{lcccc}
\hline SSS $^{\prime}$ & $\eta_{j}{ }^{\prime}(\mathrm{mm})$ & Mass, $m_{i}{ }^{\prime}(\mathrm{kg})$ & $(G t)_{\mathrm{EFF}}{ }^{\prime}\left(\mathrm{N} \cdot \mathrm{mm}^{-1}\right)$ & {$\left[S_{j} /(\mathrm{Gt})_{\mathrm{EFF}}\right]^{\prime}\left(\mathrm{mm}{ }^{3} \cdot \mathrm{N}\right)$} \\
\hline SSS-1 & 1.3103 & 8.53 & 3074.74 & 172.50 \\
SSS-2 & 1.0625 & 24.24 & 6156.18 & 252.94 \\
SSS-3 & 1.2891 & 49.20 & 4085.76 & 471.86 \\
SSS-4 & 1.0625 & 24.24 & 6156.18 & 252.94 \\
SSS-5 & 1.3103 & 8.53 & 3074.74 & 172.50 \\
SSS-6 & 1.3567 & 42.32 & 1058.15 & 1413.79 \\
SSS-7 & 0.2177 & 54.18 & 251820.89 & 5.9368 \\
SSS-8 & 0.2178 & 54.18 & 251820.89 & 5.9368 \\
\hline
\end{tabular}

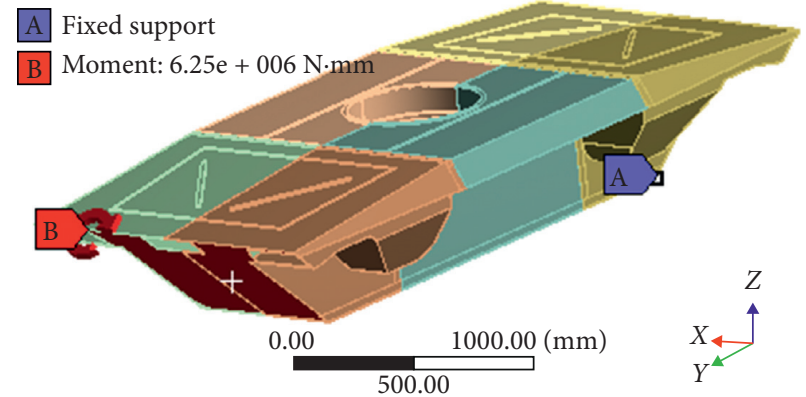

(a)

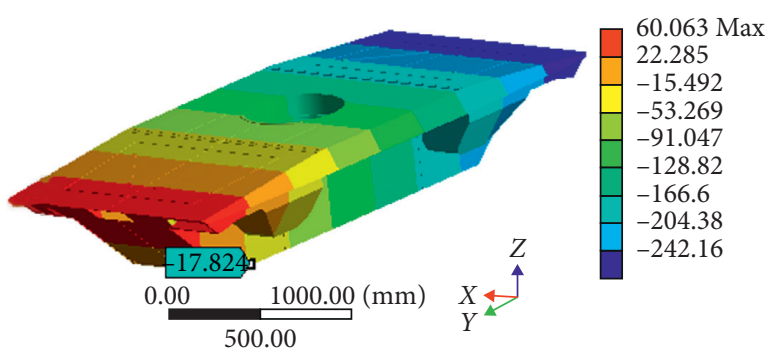

(b)

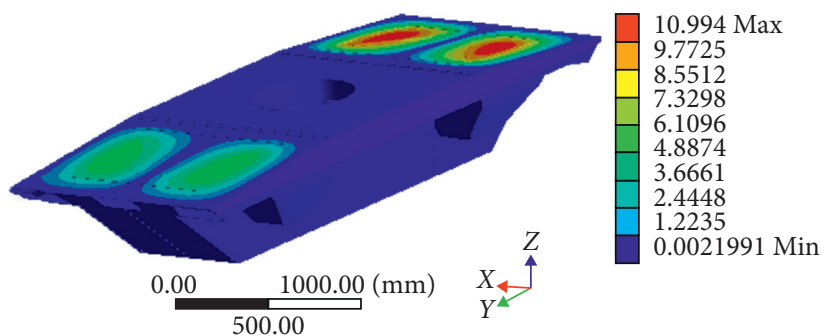

(c)

FIgURE 12: The results of BIW after optimization. (a) The boundary conditions of BIW after optimization. (b) The torsional displacement after optimization. (c) The first-order torsional frequency after optimization.

TABle 5: The comparison between the proposed method and FEM after the optimizations.

\begin{tabular}{lcc}
\hline Method & Torsional stiffness $\left(\mathrm{N} \cdot \mathrm{m} /\left(^{\circ}\right)\right)$ & First-order torsional frequency $(\mathrm{Hz})$ \\
\hline FEM & 6733.394 & 24.752 \\
Proposed method & 7139.785 & 24.987 \\
Relative error (\%) & 6.04 & 0.95 \\
\hline
\end{tabular}

It is seen that the relative error is $6.04 \%$ between the proposed square box model and the FEM model in predicting the torsional stiffness. And the relative error is $0.95 \%$ between the two models in predicting the first-order torsional frequency, indicating a high precision of the proposed square box model.

The prediction values of the square box model on the torsional stiffness and the first-order torsional frequency are larger than those of the FEM simulation. This may be caused by the simplification of the square box model, where the connection to the various structural panels is rigid. However, there is a flexible hinge which is connected with SSS panels. If flexible hinge connections were assumed, they would definitely further reduce the error and improve the calculation accuracy of the model.

\section{Conclusions}

For the existing problem of a complicated operation and high calculation cost to assess the properties of a BIW, a novel prediction method, which is collaborative square box model and MOGA, is presented to assess and optimize the properties of the BIW. Moreover, a BIW of UGV is used to illustrate the application procedure and the effectiveness of the proposed method is verified via FEM. One advantage of the square box model is that it builds an eight-DOF series 
spring system which is related to the torsional stiffness and the first-order torsional frequency. At the same time, the square box model avoids building complicated boundary conditions that are practical for actual engineering. The other great advantage is that it contributes to the size optimization of SSS to achieve the lightweight of the structure. The novel optimal strategy is applied to optimize the thickness of SSS by using MOGA within the framework of the square box model. In addition, LHS is used to represent the input parameters. Then, the response surface method is adopted to build accurate models in the parameter ranges. With the help of the square box model, it is convenient to find the weak panels of small rigidity of the BIW and to make effective structural improvement on the SSS. The results of numerical simulations demonstrate that a difference between the proposed square box model and the FEM in predicting a torsional property is less than 6.04\%, and the optimal values of thickness are reduced by around $9.9 \%$ without affecting their performance. The proposed method is verified and takes on better optimization performance.

In the coming research, different optimization methods can be collaborative to optimize the structure to further reduce the thickness of the panels for better lightweight performance. Furthermore, a physical prototype will be tested to verify the optimal result in the future.

\section{Data Availability}

No additional data are available for this paper.

\section{Conflicts of Interest}

The authors declare that they have no conflicts of interest regarding the publication of this paper.

\section{Acknowledgments}

The authors thank their colleagues who provided insight and expertise that greatly assisted with the research. This work was supported by the Key Technology of Intelligent Garden Sanitation Robot for Industrial Application (no. AA19254019).

\section{Supplementary Materials}

Figure S1: the final results of SSS-7 after optimization in the form of nephogram graph. The displacement of SSS-7 and the first-order frequency of SSS-7 can be directly obtained by the nephogram analysis. Figure S2: the complete analytical results of simple structural surfaces after the optimizations. It shows the displacement of deformation for each surface which is the supplementary material for Table 4 in Section 3.3. (Supplementary Materials)

\section{References}

[1] W. Wang, C. Xu, Z. Wang et al., "A study on finite element analysis and structural assessment method for aluminumalloy vehicle body," Automotive Engineering, vol. 6, no. 41, pp. 607-614, 2019.
[2] S. N. Ashurkova, V. V. Kobishchanov, and E. V. Kolchina, "Methods used to analyze the impact of passenger cars bodies design features on their stiffness and strength characteristics," Procedia Engineering, vol. 206, pp. 1623-1628, 2017.

[3] B. T. Chandru and P. M. Suresh, "Numerical and experimental analysis of passenger car floor," Materials Today: Proceedings, vol. 5, no. 10, pp. 22303-22311, 2018.

[4] J. Pawlowski and H. Guy, Vehicle Body Engineering, Business Book Limited, London, UK, 6th edition, 1969.

[5] H. Nishigaki, S. Nishiwaki, T. Amago et al., "First order analysis-new cae tools for automotive body designers," SAE International, Warrendale, PA, USA, SAE Technical Paper, 2001.

[6] N. Lyu and K. Saitou, "Decomposition-based assembly synthesis of a three-dimensional Body-in-White model for structural stiffness," Journal of Mechanical Design, vol. 127, no. 1, pp. 34-48, 2005.

[7] D. Mundo, R. Hadjit, S. Donders et al., "Simplified modelling of joints and beam-like structures for BIW optimization in a concept phase of the vehicle design process," Finite Elements in Analysis and Design, vol. 45, no. 6-7, pp. 456-462, 2009.

[8] W. Wang, M. Luo, W. Guo et al., "Structure dynamics analysis for use in noise reduction of an automotive body-in-white," in Proceedings of the International Conference on Information Engineering \& Computer Science, December 2009.

[9] S. Donders, Y. Takahashi, R. Hadjit et al., "A reduced beam and joint concept modeling approach to optimize global vehicle body dynamics," Finite Elements in Analysis and Design, vol. 45, no. 6-7, pp. 439-455, 2009.

[10] N. Nariman-Zadeh, M. Salehpour, A. Jamali, and E. Haghgoo, "Pareto optimization of a five-degree of freedom vehicle vibration model using a multiobjective uniform-diversity genetic algorithm (MUGA)," Engineering Applications of Artificial Intelligence, vol. 23, no. 4, pp. 543-551, 2010.

[11] Z. L. Gao, Q. H. Kong, J. P. Lin, Q. S. Hu, and Y. Chen, "A new design method of body-in-white lightweight considering NVH performance," Advanced Materials Research, vol. 482484, pp. 1979-1984, 2012.

[12] Y.-J. Cha, A. K. Agrawal, Y. Kim, and A. M. Raich, "Multiobjective genetic algorithms for cost-effective distributions of actuators and sensors in large structures," Expert Systems with Applications, vol. 39, no. 9, pp. 7822-7833, 2012.

[13] Y. Li, H. Li, Z. Pan, and T. Xu, "Development of concept analysis and multiobjective optimization platform for Bodyin-White structure," Lecture Notes in Electrical Engineering, vol. 195, pp. 361-371, 2013.

[14] D. F. Wang, F. Ji, S. M. Chen, Y. S. Li, H. B. Chen, and X. M. Zhao, "Implicit parameterization modeling and validation for body-in-white of a car," Applied Mechanics and Materials, vol. 365-366, pp. 429-434, 2013.

[15] N. F. Nangolo, J. Soukup, L. Rychlíková, and J. Skočilas, “A combined numerical and modal analysis on vertical vibration response of railway vehicle," Procedia Engineering, vol. 96, pp. 310-319, 2014.

[16] K. L. Vasudev, R. Sharma, and S. K. Bhattacharyya, "A multiobjective optimization design framework integrated with CFD for the design of AUVs," Methods in Oceanography, vol. 10, pp. 138-165, 2014.

[17] M. Kiani, H. Shiozaki, and K. Motoyama, "Simulation-based design optimisation to develop a lightweight Body-in-White structure focusing on dynamic and static stiffness," International Journal of Vehicle Design, vol. 67, no. 3, pp. 219-236, 2015. 
[18] I. P. Mitseas, I. A. Kougioumtzoglou, and M. Beer, “An approximate stochastic dynamics approach for nonlinear structural system performance-based multiobjective optimum design," Structural Safety, vol. 60, pp. 67-76, 2016.

[19] H. Qin, Z. Liu, Y. Liu, and H. Zhong, "An object-oriented MATLAB toolbox for automotive body conceptual design using distributed parallel optimization," Advances in Engineering Software, vol. 106, pp. 19-32, 2017.

[20] C. Shan, Y. Li, and W. Hou, "Optimization of car body assembly structure based on modular design," Automotive Engineering, vol. 6, no. 40, pp. 617-624, 2018.

[21] K. K. Dama, V. S. Babu, and R. N. Rao, "State of the art on automotive lightweight body-in-white design," Materials Today: Proceedings, vol. 5, no. 10, pp. 20966-20971, 2018.

[22] H. Qin, Y. Guo, Z. Liu, Y. Liu, and H. Zhong, "Shape optimization of automotive body frame using an improved genetic algorithm optimizer," Advances in Engineering Software, vol. 121, pp. 235249, 2018.

[23] N. A. Zolpakar, S. S. Lodhi, S. Pathak et al., "Application of multiobjective genetic algorithm (moga) optimization in machining processes," Optimization of Manufacturing Processes, Springer, Berlin, Germany, 2019.

[24] W. Deng, J. Xu, Y. Song et al., "Differential evolution algorithm with wavelet basis function and optimal mutation strategy for complex optimization problem," Applied Soft Computing, 2020, In press, Article ID 106724.

[25] W. Deng, H. Liu, J. Xu, H. Zhao, and Y. Song, "An improved quantum-inspired differential evolution algorithm for deep belief network," "IEEE Transactions on Instrumentation and Measurement, vol. 69, no. 10, pp. 7319-7327, 2020.

[26] W. Deng, J. Xu, H. Zhao, and Y. Song, "A novel gate resource allocation method using improved PSO-based QEA," IEEE Transactions on Intelligent Transportation Systems, pp. 1-9. In press, 2020.

[27] N. Ferro, S. Micheletti, and S. Perotto, "An optimization algorithm for automatic structural design," Computer Methods in Applied Mechanics and Engineering, vol. 372, Article ID 113335, 2020.

[28] S. Li and X. Feng, "Study of structural optimization design on a certain vehicle Body-in-White based on static performance and modal analysis," Mechanical Systems and Signal Processing, vol. 135, Article ID 106405, 2020.

[29] E. Bonisoli, D. Lisitano, and A. Vigliani, "Damping identification and localisation via Layer Method: experimental application to a vehicle chassis focused on shock absorbers effects," Mechanical Systems and Signal Processing, vol. 116, no. 2, pp. 194-216, 2019.

[30] I. Gholaminezhad, A. Jamali, and H. Assimi, "Multiobjective reliability-based robust design optimization of robot gripper mechanism with probabilistically uncertain parameters," Neural Computing and Applications, vol. 28, no. S1, pp. 659-670, 2017. 